\title{
Comprehensive Circular RNA Profiling Reveals the Regulatory Role of the CircRNA-0067835/miR-155 Pathway in Temporal Lobe Epilepsy
}

\author{
Guo-Hua Gonga,beng-Mao An ${ }^{a, b}$ Yu Wang ${ }^{a, b}$ Ming Bian ${ }^{a, b}$ \\ Di Wanga,b Cheng-Xi Wei ${ }^{a, b}$
}

aMedicinal Chemistry and Pharmacology Institute, Inner Mongolia University for the Nationalities, Tongliao, Inner Mongolia, ' Inner Mongolia Key Laboratory of Mongolian Medicine Pharmacology for Cardio-Cerebral Vascular System, Tongliao, Inner Mongolia, 'First Clinical Medical of Inner Mongolia University for Nationalities, Tongliao, Inner Mongolia, China

\section{Key Words}

Epilepsy $\bullet$ TLE $・$ Circular RNA $・$ microRNA $・$ Sponge

\begin{abstract}
Background/Aims: Temporal lobe epilepsy (TLE) is the most common form of adult localization-related epilepsy that is accompanied by progressive etiopathology and high incidences of drug resistance. Circular RNAs (circRNAs) play important roles in fine-tuning gene expression, however, the expression profile and clinical significance of circRNAs in TLE remains unknown. Methods: Circular RNA microarray was conducted to identify TLE-related circRNAs. CCK8 assays and flow cytometric assays were conducted to clarify the role of circRNA in TLE in vitro. Bioinformatics analysis and in vitro experiments were conducted to clarify the mechanism of circRNA-mediated gene regulation in TLE cell. Results: 586 differentially expressed circRNAs were identified between TLE and the control tissues. The expression of circRNA-0067835 was significantly down-regulated in tissues and plasma from TLE patients. Lower circRNA-0067835 correlated to increased seizure frequency, HS, and higher Engel's score. Overexpression of circRNA-0067835 observably decreased SH-SY5Y cell proliferation by causing $\mathrm{G} 1$ arrest and promoting apoptosis. Bioinformatics online programs predicted that circRNA-0067835 acted as miR-155 sponge to regulate FOXO3a expression, which was validated using luciferase reporter assay. Conclusion: Our experiments showed that circRNA-0067835 regulated refractory epilepsy progression by acting as a sponge of miR-155 to promote FOXO3a expression, indicating that circRNA-0067835 may serve as a potential therapeutic target for patients with TLE.

G. Gong and F. An contributed equally to this work.




\section{Cellular Physiology Cell Physiol Biochem 2018;51:1399-1409

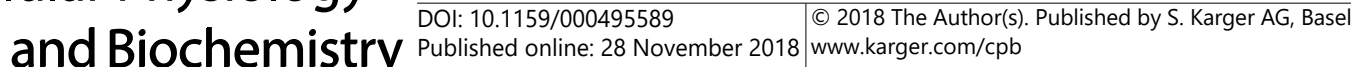

Gong et al.: CircRNA-0067835/miR-155 Pathway in Temporal Lobe Epilepsy

\section{Introduction}

Epilepsy is a chronic brain disease characterized by the presence of recurrent seizures, which are the result of an excessive electrical discharge of a neuronal group in a certain part of the brain [1-2]. Currently, antiepileptic drugs (AEDs) merely provide symptomatic control of seizures. Resistance to AEDs has been an important clinical challenge in refractory epilepsy therapy for neurologists [3-4]. Temporal lobe epilepsy (TLE) is the most common form of adult localization-related epilepsy that is accompanied by progressive etiopathology and high incidences of drug resistance [5-6]. While great progress has been made in the past decades in understanding of the neuropathology of TLE, the molecular mechanisms underlying pathogenic and drug resistance of this disorder remain poorly understood. So it is quite critical to find new treatment options to improve drug resistance for TLE.

Circular RNAs (circRNAs) are recently identified as novel class of endogenous noncoding RNAs (ncRNAs) that is highly represented in the eukaryotic transcriptome [7-8]. CircRNAs could regulate the gene expression at the transcriptional or post-transcriptional level by acting as microRNA (miRNA) sponges [8-9]. Recently, circRNAs have been reported to be upregulated during mouse neural development and human epithelial-mesenchymal transition [10]. However, the biological functions of circRNAs in human diseases, especially in TLE, are unclear. In this study, based on circRNA microarray analysis, we focused on the role of circRNA-0067835 in TLE. Our experiments showed that circRNA-0067835 regulated refractory epilepsy progression by acting as a sponge of miR-155 to promote FOXO3a expression, indicating that circRNA-0067835 may serve as a potential therapeutic target for TLE.

\section{Materials and Methods}

\section{Patient selection and tissue collection}

Temporal cortex tissues resected from 22 patients with drug-resistant TLE who had undergone anterior temporal lobectomy were collected. The 22 control samples of temporal neocortical tissues without abnormal pathological changes were obtained. None of patients in control group was ever diagnosed of epilepsy or seizures. Resected brain tissues were immediately frozen in liquid nitrogen for further studies. Five pairs of TLE samples and control samples were used for circRNA microarray. Subsequently, a total of 22 paired samples, including the samples for microarray analysis, were used for circRNA validation using reverse transcriptase quantitative PCR. For miRNA expression analysis, small RNAs from temporal cortex tissue from patients or controls were isolated using miRNA extraction kit (Qiagen) according to manufacture's instruction. For detection of FOXO3a, RNA was extracted according to Trizol method (Invitrogen, USA). This study was approved by the Human Research Ethics Committee from Inner Mongolia University for the Nationalities. All human subject research was performed in accordance with institutional, national, and Declaration of Helsinki requirements, and before analysis, written informed consent was obtained from all patients.

\section{Cell Culture}

Human neuroblastoma SH-SY5Y cells and human embryonic kidney 293 cells (HEK293) were provided by the cell resource center of Chinese academy of medical sciences (Beijing, China). Cells were cultured in Dulbecco's modified Eagle's medium (DMEM) (Invitrogen, Carlsbad, CA, USA) containing $10 \%$ heatinactivated fetal bovine serum (FBS; Invitrogen), 100 units $/ \mathrm{ml}$ penicillin, and $100 \mu \mathrm{g} / \mathrm{mL}$ of streptomycin (Sigma-Aldrich, St. Louis, USA) in a $37^{\circ} \mathrm{C}$ and $5 \% \mathrm{CO} 2$ incubator.

\section{Expression profile analysis of circRNAs}

Five pairs of TLE samples and control samples were used for circRNA microarray. Tissues specimens were obtained during operation and immediately frozen at $-80^{\circ} \mathrm{C}$ until further use. The circRNAs chip (ArrayStar Human circRNAs chip; ArrayStar, Rockville, MD, USA) containing 5, 639 probes specific for human circular RNAs splicing sites was used. Following hybridization and washing of the samples, 5 pairs 


\section{Cellular Physiology Cell Physiol Biochem 2018;51:1399-1409

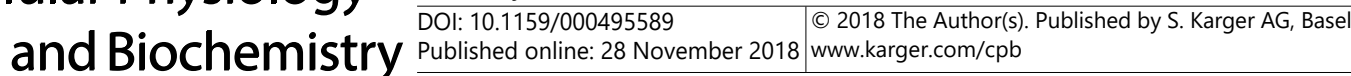 \\ Gong et al.: CircRNA-0067835/miR-155 Pathway in Temporal Lobe Epilepsy}

of TLE samples and control samples were analyzed on the circRNAs chips. Exogenous RNAs developed by the External RNA Controls Consortium (ERCC) were used as controls. circRNAs were enriched by digesting linear RNA with RNase R (Epicentre, Madison, WI, USA). Labeled RNAs were scanned using a Agilent Scanner G2505C (Agilent Technologies, Santa Clara, CA, USA). The circRNA microarray process was performed by KangChen Biotech, Inc. (Shanghai, China).

\section{Plasma collection and RNA Extraction}

$5 \mathrm{ml}$ blood samples from all TLE cases and surgical control cases were collected in EDTA-containing tubes. Whole-blood samples were centrifuged at $1800 \mathrm{~g}$ for $20 \mathrm{~min}$ at $4{ }^{\circ} \mathrm{C}$ after blood collection, and the upper plasma phase was carefully transferred into microcentrifuge tube. A second centrifugation at 1600 $\mathrm{g}$ for $10 \mathrm{~min}$ at $4{ }^{\circ} \mathrm{C}$ to remove additional cellular debris and minimize contamination of cell-free nucleic acids derived from damaged blood cells. For long storage, plasma frozen in aliquots was kept at $-80{ }^{\circ} \mathrm{C}$ until further analysis. Total RNA was extracted from $100 \mu \mathrm{l}$ of plasma samples using TRIzol LS reagent (Life Technologies) according to manufacturer's instructions.

\section{Quantitative Real-Time PCR}

Total RNA and miRNAs were isolated using Trizol reagent (Invitrogen) and mirVana miRNA isolation kit (Ambion, Austin, TX, USA). Subseequently, cDNA was synthesized and amplified by RT-qPCR based on the TaqMan method on an ABI PRISM 7500 Sequence Detection System (Life Technologies, Grand Island, NY, USA) with the housekeeping gene GAPDH or U6 as an internal control. Relative expression levels of the genes were calculated using the $2^{-\Delta \Delta \mathrm{Ct}}$ method.

\section{Bioinformatics analysis.}

The online target-predicting database miRBase (http://www.mirbase.org/) was used for the prediction of potential targeted sequences between circRNA-0067835 and miR-155. Another two databases, TargetScan (http://www.targetscan.org/) and miRanda (http://www.microrna.org/microrna/home.do), were used for the prediction of potential targeted sequences between miR-155 and the FOXO3a gene.

\section{Cell transfection}

The miR-155 mimics, and specific anti-miR-155 RNAs were synthesized by GenePharma (Shanghai, China). The circular transcript expression vector possesses two elements termed as the front circular and the back circular frame which were specially designed containing inverted repeat sequences flank. The full-length cDNA of circRNA-0067835 was amplified into cells, and was cloned into the specific vector between two frames, while the mock plasmid without the circRNA-0067835 cDNA served as the control. The cDNA sequence of circRNA- 0067835 was synthesized by RiboBio Co., Ltd. (Guangzhou, China) and then cloned into the lentiviral expression vector, pLVXIRES-neo (Clontech Laboratories Inc., San Francisco, CA, USA). Lentiviral production and transduction were conducted by following previously published procedures. Cells were maintained in a 6-well plate in DMEM supplemented with 10\% FBS and cultured until 50-70\% confluent. RNA oligoribonucleotides were mixed with Lipofectamine 3000 (Invitrogen) in reduced serum medium (Opti-MEM; Gibco, Carlsbad, CA, USA) according to the manufacturer's instructions and the final concentration of the RNA oligoribonucleotides was $100 \mathrm{nM}$. The effects of knockdown or overexpression were examined by RT-qPCR using RNA extracted $48 \mathrm{~h}$ after transfection.

\section{Cell proliferation assay}

Cell proliferation assays were conducted using the Cell Counting Kit-8 (CCK-8) (Dojindo, Kumamoto, Japan) according to the manufacturer's instructions. In brief, transfected cells were seeded into 96-well plates and cultured for $0,24,48$, and $96 \mathrm{~h}$. Then, $10 \mu \mathrm{L}$ of CCK-8 solution was added to each well, and the plates were incubated at $37^{\circ} \mathrm{C}$ for an additional $2 \mathrm{~h}$. Finally, the solution was measured at $450 \mathrm{~nm}$ on the spectrophotometer (EnSpire; PerkinElmer Inc., Waltham, MA, USA). 


\section{Cellular Physiology Cell Physiol Biochem 2018;51:1399-1409

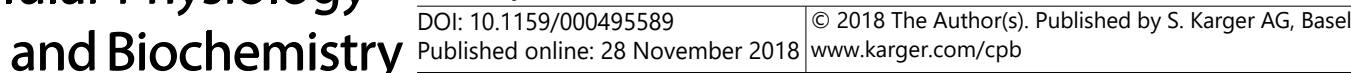 \\ Gong et al.: CircRNA-0067835/miR-155 Pathway in Temporal Lobe Epilepsy}

Flow cytometric analysis

Cells were harvested directly or $48 \mathrm{~h}$ after siRNA transient transfection and washed with ice-cold phosphate-buffered saline (PBS). The annexin V-fluorescein isothiocyanate (FITC) apoptosis detection kits (KeyGEN Biotech, Nanjing, China) was used to detect apoptosis in a FACScan instrument (Becton Dickinson, Mountain View, CA, USA), respectively.

\section{Western blot analysis}

Lysate protein from tissue or cells was assayed by BCA (Pierce), and equal amounts of lysate protein were loaded on to BisTris XT denaturing 10\% polyacrylamide gels containing SDS (Bio-Rad Laboratories). Proteins were resolved by SDS/PAGE and transferred on to PVDF membranes. The samples were blocked and then incubated with the primary antibody anti-FOXO3a diluted at 1:1, 000 (Abcam, USA) at $4^{\circ} \mathrm{C}$ overnight. The membranes were washed and then incubated with HRP-labeled goat anti-mouse secondary antibody $(1: 10,000$, Pierce, USA) at room temperature for $1 \mathrm{~h}$, separately. The bands were detected using a chemiluminescent substrate (Millipore, USA). The GAPDH was used as an internal control. Gray values were measured using ImageJ Analysis Software (NIH). The relative expressions of samples in different duplications were standardized by a same sample of control group.

\section{Luciferase activity assays}

Cells were co-transfected with circRNA-0067835 plasmids or their mutant fragments and miR-155 mimic by using Lipofectamine 2000 (Invitrogen, Foster city, CA) according to the manufacturer's protocol. Firefly and Renilla luciferase activities were measured consecutively using a Dual-Luciferase Reporter Assay System (Promega, Massachusetts, USA) after transfection for $48 \mathrm{~h}$. Each assay was repeated in six independent experiments.

\section{Statistical Analysis}

All data were normally distributed and presented as mean values \pm s.e. or s.d. as specified. Statistical differences between two groups were evaluated by two tailed unpaired Student's t-test or with a two-sided Mann Whitney test with $t$ approximation. In the case of multiple mean comparisons one-way analysis of variance (ANOVA) was used (Gnumeric 1.12.12; SPSS Statistics 19, IBM, USA). P-values $<0.05$ were regarded as significant.

\section{Results}

\section{Analysis of circRNA expression profiles in human TLE}

To identify specific circRNAs that are differentially expressed between patients with drug-resistant TLE and control samples of temporal neocortical tissues, 5 TLE samples and control samples were subjected for circRNA microarray assay. A total of 586 circRNAs that were differently expressed by $>3$ fold between the TLE groups and the control tissues were identified. Subsequently, we narrowed the scope of the analysis to the 30 most aberrantly expressed circRNAs, including 15 upregulated circRNAs and 15 downregulated circRNAs (shown in the heatmap in Fig. 1A). We selected 10 mostly dysregulated circRNAs for validation using real-time PCR in 22 paired of samples. General consistency was shown between the highthroughput data and real-time PCR results, we observed significantly reduced expression of circRNA-0067835 in tissues of 22 surgical patients with TLE patients as compared with controls (Fig. 1B; $P<0.01$ ).

\section{Relationship between circRNA-0067835 in plasma and clinical features in patients with}

TLE

Consistent with data from brain tissue, we observed significantly reduced expression of circRNA-0067835 in plasma of 22 surgical patients with TLE patients as compared with controls (Fig. 1C; $P<0.01$ ). Based on these results, we further evaluated the association between the circRNA-0067835 in plasma and the clinical features in patients with TLE in order to better understand its potential role in the progression of TLE. We revealed that 
lower circRNA-0067835 correlated to increased seizure frequency, HS, and higher Engel's score. Taken together, these results suggested that circRNA-0067835 might play a role in the progression of TLE.

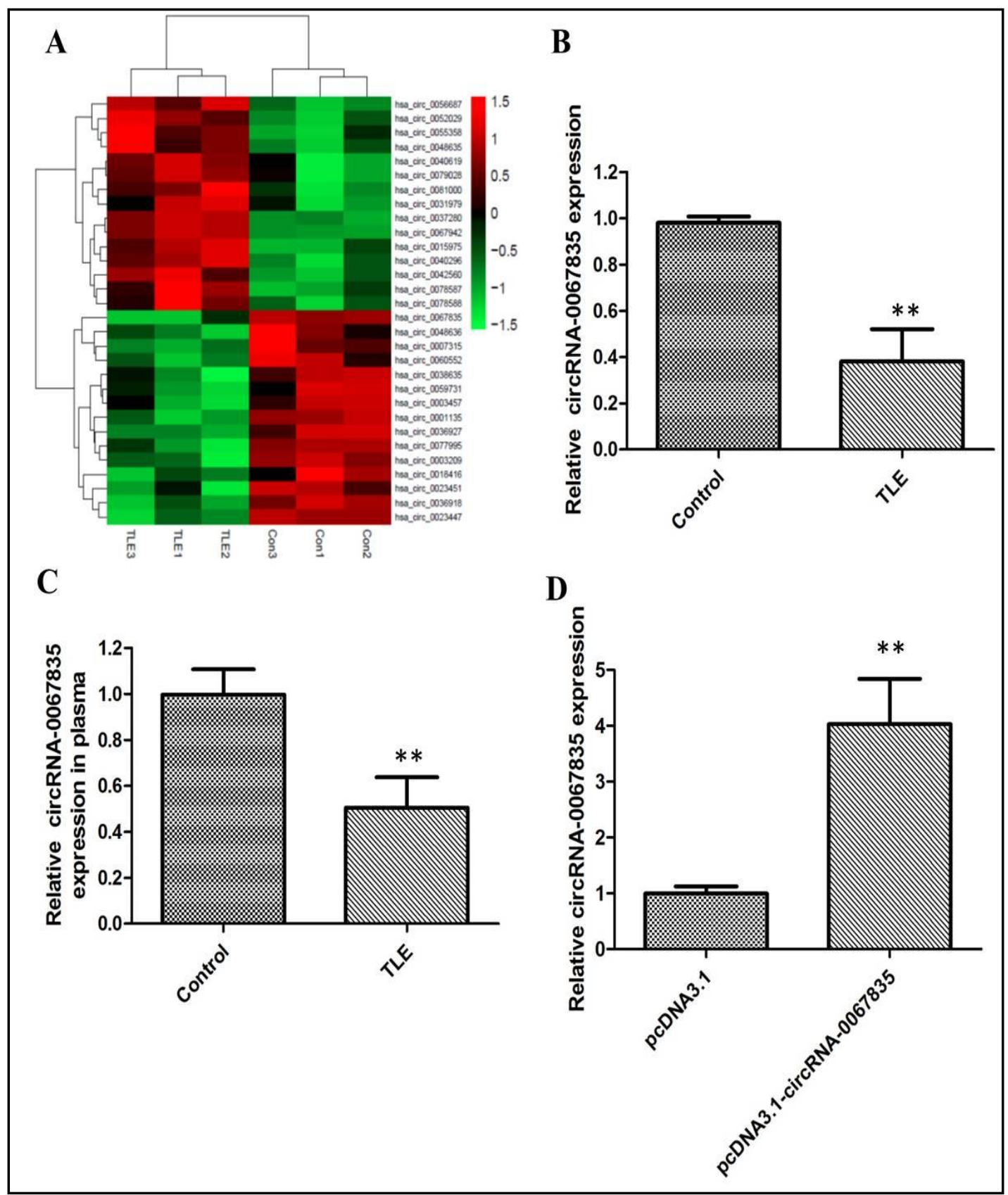

Fig. 1. (A) Differential expression of selected circRNAs in TLE and control samples of temporal neocortical tissues. Based on the circRNA microarray results, the top 15 upregulated and top 15downregulated circRNAs in TLE compared with control samples are shown in the heatmap. The red colour represents high expression, whereas the green colour represents low expression. (B) qRT-PCR verification of the expression of circRNA-0067835 in tissues of 22 surgical patients with TLE patients as compared with controls. (C) qRTPCR verification of the expression of circRNA-0067835 in plasma of 22 surgical patients with TLE patients as compared with controls. (D) The qRT-PCR results presented that circRNA-0067835 expression in SHSY5Y cells was remarkably increased after transfection with pcDNA3.1-circRNA-0067835. All tests were at least performed three times. Data were expressed as mean \pm SD. ${ }^{* *} \mathrm{P}<0.01$. 
A

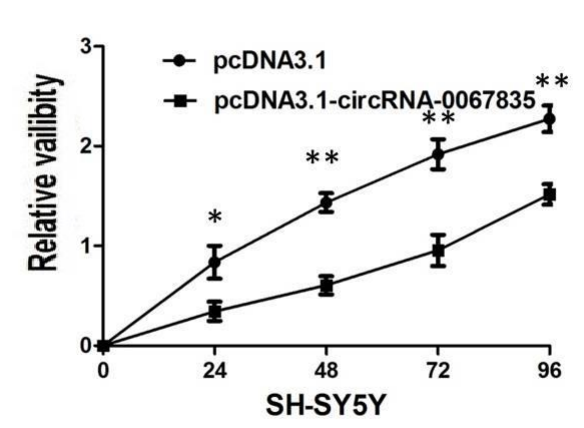

C

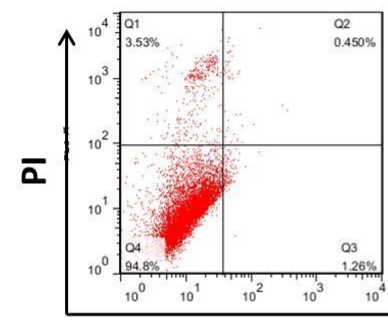

Annexin V

pcDNA3.1

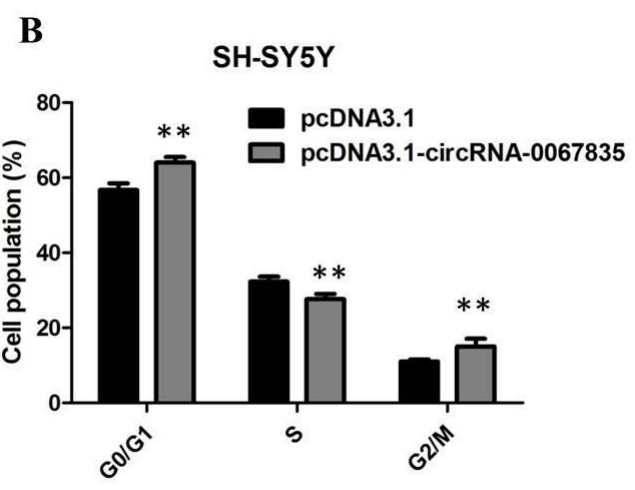

D
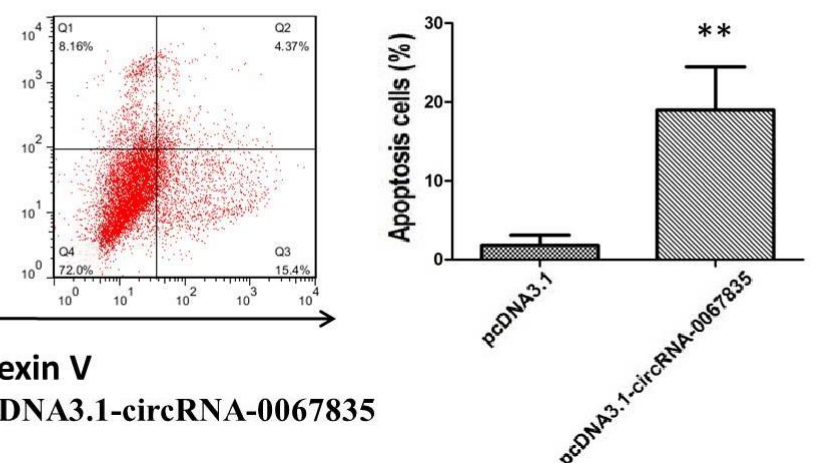

Fig. 2. (A) CCK8 assay showing overexpression of circRNA-0067835 observably suppressed SH-SY5Y cell proliferation. (B) The flow cytometry assay showed that overexpression of circRNA-0067835 had cell-cycle arrest at the G1-G0 phase in SH-SY5Y cells. (C-D) The flow cytometry assay showed that overexpression of circRNA-0067835 had higher apoptotic rate in SH-SY5Y cells. All tests were at least performed three times. Data were expressed as mean $\pm \mathrm{SD} .{ }^{*} \mathrm{P}<0.05,{ }^{* *} \mathrm{P}<0.01$.

\section{Effects of circRNA-0067835 on SH-SY5Y cells}

The qRT-PCR results presented that circRNA-0067835 expression in SH-SY5Y cells was remarkably increased after transfection with pcDNA3.1-circRNA-0067835 (Fig. 1D, $P$ $<0.01$ ). Furthermore, CCK-8 assay showed overexpression of circRNA-0067835 observably suppressed SH-SY5Y cell proliferation (Fig. 2A, $P<0.01$ ). In addition, flow cytometry analysis disclosed the variance of cell cycle and apoptosis condition after transfection with pcDNA3.1-circRNA-0067835. As exhibited in Fig. 2B, the percentage of cells arrested in G0/ G1 phase was significantly higher in pcDNA3.1-circRNA-0067835 group than in NC group, while that of arrested cells in S phase relatively declined compared with NC group $(P<0.01)$. Meanwhile, the apoptosis rate of SH-SY5Y cells significantly increased after transfection (Fig. $2 \mathrm{C}$ and $\mathrm{D}, P<0.01$ ). Overall, overexpression of circRNA-0067835 arrested cell propagation and cell cycle and promoted apoptosis in SH-SY5Y cells.

CircRNA-0067835 functions as a miRNA sponge for miR-155 in TLE

As circRNAs function mainly as miRNA sponges to bind to functional miRNAs and then regulate gene expression, we then examined the potential miRNAs associated with circRNA-0067835. According to bioinformatics analysis using miRBase (http://www. 
A

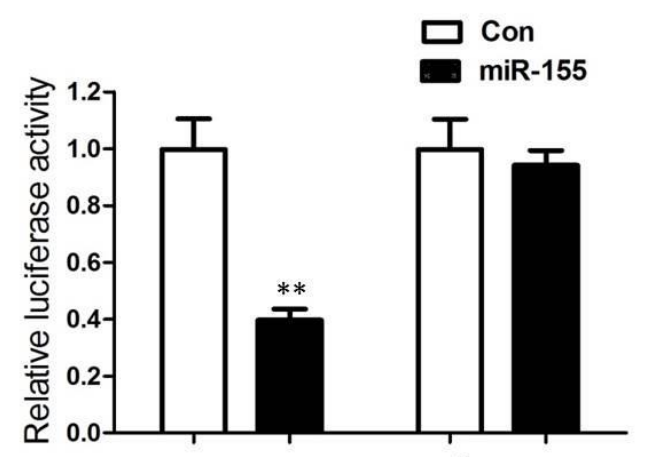

WT-circRNA-0067835 MUT-circRNA-0067835

C

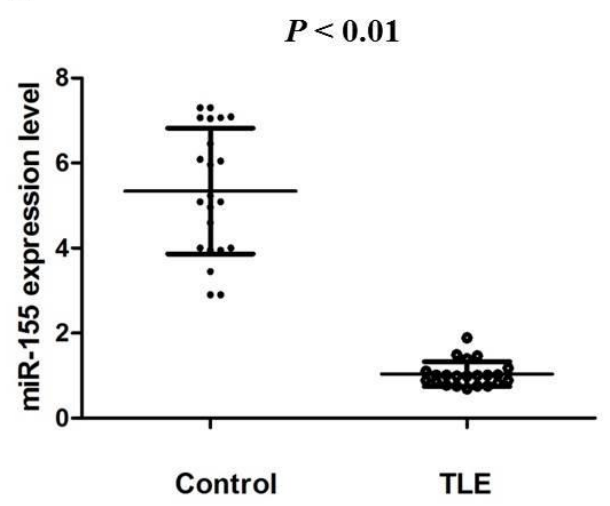

\section{B}
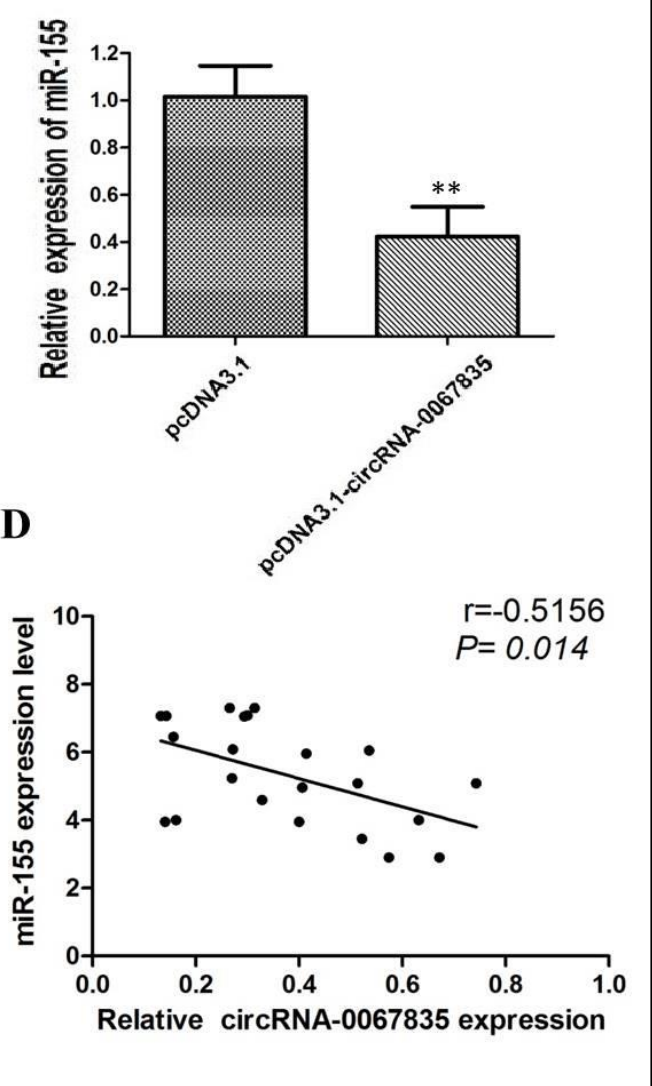

Fig. 3. (A) The luciferase activity was repressed in SH-SY5Y cells co-transfected with wild-type (WT) circRNA-0067835 and miR-155 mimics while restored in cells cotransfected with mutant (Mut) circRNA-0067835 and miR-155 mimics. (B) Overexpression of circRNA-0067835 significantly suppressed miR-155 expression in SH-SY5Y cells. (C) The qRT-PCR results presented that miR-155 expression was remarkably decreased in TLE compared with control samples. (D) There was an inverse correlation between the levels of circRNA-0067835 and miR-155 in drug-resistant TLE. All tests were at least performed three times. Data were expressed as mean $\pm \mathrm{SD} .{ }^{*} \mathrm{P}<0.05$, ${ }^{* *} \mathrm{P}<0.01$.

mirbase.org/), circRNA-0067835 possessed a complementary sequence to the miR-155 seed region. To confirm whether circRNA-0067835 directly binds to miR-155, we performed dualluciferase reporter assay. The results displayed that luciferase activity was repressed in SHSY5Y cells co-transfected with wild-type (WT) circRNA-0067835 and miR-155 mimics while restored in cells cotransfected with mutant (Mut) circRNA-0067835 and miR-155 mimics (Fig. 3A). Moreover, enhanced expression of circRNA-0067835 significantly suppressed miR-155 expression in SH-SY5Y cells (Fig. 3B). MiR-155 expression was also studied in in 22 paired of tissue samples, and the results of RT-qPCR also revealed that a statistically decrease compared to temporal cortex tissues from controls $(P<0.01$, Fig. 3C). There was an inverse correlation between the levels of circRNA-0067835 and miR-155 in drug-resistant TLE (R=-0.5156, $P=0.014$, Fig. 3D). These data suggested miR-155was a direct target of circRNA-0067835 in TLE.

miR-155 exerts its role by regulation of FOXO3a expression in TLE

We further explored the mechanism by which miR-155 participated in TLE, potential target genes for miR-155 were therefore searched for using bioinformatics software. On the basis of miRNA target analysis, FOXO3a is a potential target mRNA of miR-155 (Fig. 4A). To 


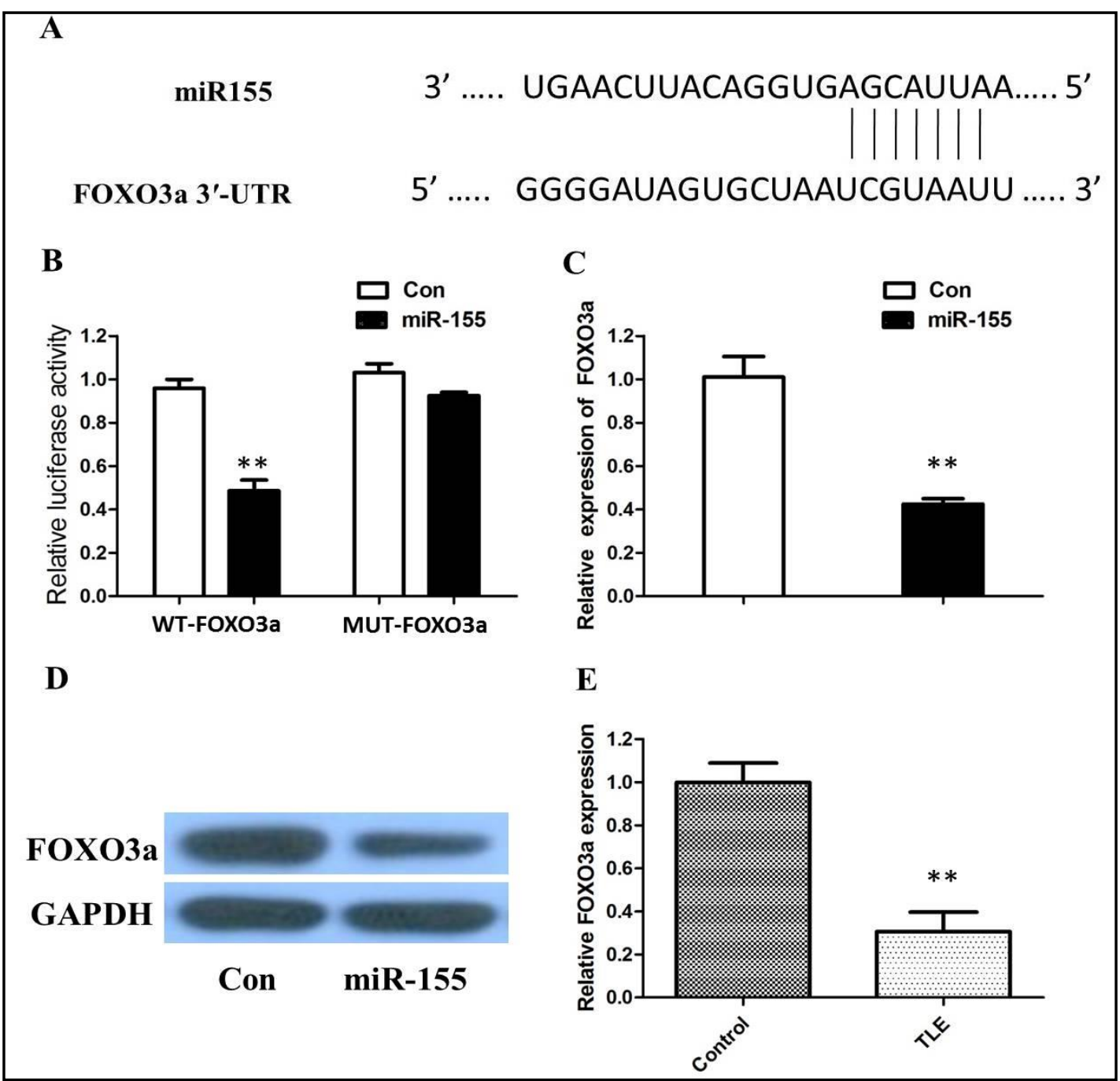

Fig. 4. (A) Bioinformatics analysis revealed the predicted binding sites between FOXO3 and miR-155. (B) Luciferase reporter assay demonstrated miR-155 mimics significantly decreased the luciferase activity of FOX03-wt in HEK293T cells. (C) Overexpression of miR-155 inhibited the mRNA and protein levels of FOXO3a in SH-SY5Y cells. (D) The qRT-PCR results presented that FOXO3a expression was remarkably increased in TLE compared with control samples. All tests were at least performed three times. Data were expressed as mean $\pm \mathrm{SD}$. ${ }^{* *} \mathrm{P}<0.01$.

prove it, we performed luciferase reporter assay to validate the interaction between miR-155 and the 3'UTR of FOXO3a mRNA. The results indicated that luciferase activity was reduced in HEK293 cells co-transfected with FOXO3a-WT and miR-155 mimics and restored in cells cotransfected with FOXO3a-Mut and miR-155 mimics (Fig. 4B). Furthermore, we showed that overexpression of miR-155 inhibited the mRNA and protein levels of FOXO3a in SH-SY5Y cells (Fig. 4C and D). We then explored human FOXO3a mRNA level in TLE patients. As expected, the levels of human FOXO3a mRNA were reduced in temporal cortex of TLE patients (Fig. $4 \mathrm{E})$. Pearson's correlation test showed there is a negative correlation between miR-155 and FOX03a $(\mathrm{P}<0.01)$. In summary, above results indicated that miR-155 modulates FOXO3a expression in TLE. 


\section{Cellular Physiology Cell Physiol Biochem 2018;51:1399-1409

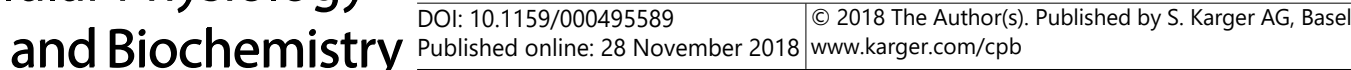 \\ Gong et al.: CircRNA-0067835/miR-155 Pathway in Temporal Lobe Epilepsy}

\section{Discussion}

In this study, we investigated the circRNA expression profile in drug-resistant TLE and control samples of temporal neocortical tissues. We found that 586 circRNAs that were differently expressed by $>3$ fold between the TLE groups and the control tissues. Specifically, we showed that downregulation of circRNA-0067835 could be an important contributor to drug-resistant TLE. We found that circRNA-0067835 was decreased in tissues and plasma of 22 surgical patients with TLE patients, while miR-155 expression was upregulated in TLE. Low expression of circRNA-0067835 correlated to increased seizure frequency, HS, and higher Engel's score in TLE patients. Moreover, overexpression of circRNA-0067835 arrested cell propagation and cell cycle and promoted apoptosis in SH-SY5Y cells. Specifically, we also showed mechanistically that circRNA-0067835 regulates the progression of TLE by inhibiting miR-155 expression. In conclusion, evidence for circRNA-0067835 functionality in TLE also extended to clinicopathological features of TLE patients, and may be considered for the development of potential therapeutics against TLE.

Currently, resective surgeries for focal epilepsy and new-generation AEDs are the major treatments for patients with epilepsy. TLE is a common type of epilepsy that is accompanied by resistance to multiple AEDs and requires surgical resection of the epileptic focus [11]. As a novel gene regulator, circRNAs are potentially involved in multiple biological and pathological processes [12-14]. However, very little is known regarding their roles in TLE. Recently, Li et al. used high-throughput sequencing methods to profile genome-wide circRNA expression levels in TLE patients and assess the role of circRNAs in human TLE. They found that dysregulations of circRNAs may reflect the pathogenesis of TLE and circ-EFCAB2 and circ-DROSHA might be potential therapeutic targets and biomarkers in TLE patients [15].

Firstly, we used the circRNA microarray assay to investigate specific circRNAs that are differentially expressed between patients with drug-resistant TLE and control samples of temporal neocortical tissues, and then we identified one circRNA, circRNA-0067835, via microarray sequences. Firstly, circRNA-0067835 was significantly downregulated in TLE tissues in contrast to control tissues. In agreement with high-throughput data, the validation array by RT-qPCR further confirmed its expression mode in TLE. We also found that reduced expression of circRNA-0067835 in plasma was significantly correlated to increased seizure frequency, HS, and higher Engel's score in patients with TLE. Moreover, in vitro functional assays showed that overexpression of circRNA-0067835 observably suppressed SH-SY5Y cell proliferation. In addition, overexpression of circRNA-0067835 promoted significant arrest in the G0/G1-phase and resulted in an obvious increase of apoptosis in SH-SY5Y cells, suggesting that circRNA-0067835 may play an important role in TLE. Despite the small number of circRNA reports in TLE studies, to our knowledge, this study is one of the first few differential expression analyses of circRNAs reported for TLE. More importantly, we identified a novel circRNA target, circRNA-0067835, for TLE clinical diagnosis and patient treatments.

Since the first report of circRNA functioning as a miRNA sponge, the potential of circRNAs in regulating cancer-related genes through fine-tuning miRNAs has recently been recognized [16-17]. In this study, miR-155 showed a complementary sequence to circRNA-0067835 based on bioinformatics analysis, and this miRNA was finally identified as the endogenous competing RNA by luciferase reporter assay. Moreover, enhanced expression of circRNA-0067835 significantly suppressed miR-155 expression in SH-SY5Y cells. As a pro-inflammatory and pro-apoptotic miRNA, miR-155 has been reportedly plays a role in the regulation of inflammatory pathways in the study of pediatric patients with TLE [18]. Recently, miR-155 is regarded as pro-epileptogenic in animals and humans with TLE [19]. We showed that the expression of miR-155 was upregulated in temporal cortex tissues samples of patients with drug-resistant TLE. There was an inverse correlation between the levels of circRNA-0067835 and miR-155 in drug-resistant TLE, indicating that circRNA-0067835 may play an anti-oncogenic role through the sponge activity of miR-155. 


\section{Cellular Physiology Cell Physiol Biochem 2018;51:1399-1409

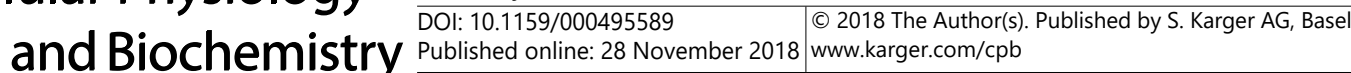 \\ Gong et al.: CircRNA-0067835/miR-155 Pathway in Temporal Lobe Epilepsy}

Finally, we sought to define the direct downstream signaling pathways that are regulated by circRNA-0067835/miR-155 pathway. In the present study, FOXO3a gene was then identified as a direct target of miR-155 by performing bioinformatics analysis and subsequent luciferase reporter assay. We found that FOXO3a was down-regulated in TLE tissues and the mRNA expression level of FOXO3a was negatively correlated with miR-155 expression, but positively correlated with HOXA-AS2 expression in TLE tissues. The luciferase assay confirmed the existence of specific crosstalk between circRNA-0067835 and FOXO3a through competition for miR-155 binding.

\section{Conclusion}

Collectively, our study demonstrated that circRNA-0067835 is an important modifier of pathogenesis of pharmacoresistant TLE by serving as a miRNA sponge for miR-155 and thereby promoting the function of FOXO3a signaling pathway. The circRNA-0067835/ miR-155/ FOXO3a regulatory network may contribute to the development of new efficient therapeutic approaches for TLE.

\section{Disclosure Statement}

The authors declare no conflicts of interest.

\section{References}

1 Rummel C, Slavova N, Seiler A, Abela E, Hauf M, Burren Y, Weisstanner C, Vulliemoz S, Seeck M, Schindler K, Wiest R: Personalized structural image analysis in patients with temporal lobe epilepsy. Sci Rep 2017;7:10883.

2 Khan FA, Poongkunran M, Buratto B: Desensitization of stimulation-induced weight loss: A secondary finding in a patient with vagal nerve stimulator for drug-resistant epilepsy. Epilepsy Behav Case Rep 2017;8:51-54.

3 Tang F, Hartz AMS, Bauer B: Drug-Resistant Epilepsy: Multiple Hypotheses, Few Answers. Front Neurol 2017;8:301.

4 Thom M: Recent advances in the neuropathology of focal lesions in epilepsy. Expert Rev Neurother 2004;4:973-984.

5 Chang BS, Lowenstein DH: Epilepsy. N Engl J Med 2003;349:1257-1266.

6 Ramey WL, Martirosyan NL, Lieu CM, Hasham HA, Lemole GM, Jr., Weinand ME: Current management and surgical outcomes of medically intractable epilepsy. Clin Neurol Neurosurg 2013;115:2411-2418.

7 Chen BJ, Byrne FL, Takenaka K, Modesitt SC, Olzomer EM, Mills JD, Farrell R, Hoehn KL, Janitz M: Analysis of the circular RNA transcriptome in endometrial cancer. Oncotarget 2018;9:5786-5796.

-8 Hansen TB, Jensen TI, Clausen BH, Bramsen JB, Finsen B, Damgaard CK, Kjems J: Natural RNA circles function as efficient microRNA sponges. Nature 2013;495:384-388.

-9 Salzman J, Chen RE, Olsen MN, Wang PL, Brown PO: Cell-type specific features of circular RNA expression. PLoS Genet 2013;9:e1003777.

$>10$ Zhou J, Xiong Q Chen H, Yang C, Fan Y: Identification of the Spinal Expression Profile of Non-coding RNAs Involved in Neuropathic Pain Following Spared Nerve Injury by Sequence Analysis. Front Mol Neurosci 2017;10:91.

11 Blumcke I, Aronica E, Miyata H, Sarnat HB, Thom M, Roessler K, Rydenhag B, Jehi L, Krsek P, Wiebe S, Spreafico R: International recommendation for a comprehensive neuropathologic workup of epilepsy surgery brain tissue: A consensus Task Force report from the ILAE Commission on Diagnostic Methods. Epilepsia 2016;57:348-358. 


\section{Cellular Physiology Cell Physiol Biochem 2018;51:1399-1409

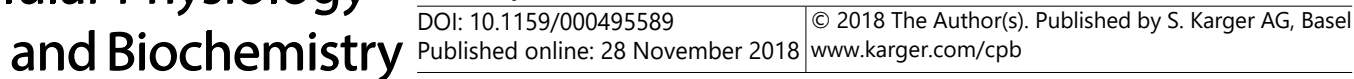

12 Wu Q, Wang Y, Cao M, Pantaleo V, Burgyan J, Li WX, Ding SW: Homology-independent discovery of replicating pathogenic circular RNAs by deep sequencing and a new computational algorithm. Proc Natl Acad Sci U S A 2012;109:3938-3943.

13 Li J, Yang J, Zhou P, Le Y, Zhou C, Wang S, Xu D, Lin HK, Gong Z: Circular RNAs in cancer: novel insights into origins, properties, functions and implications. Am J Cancer Res 2015;5:472-480.

14 Peng L, Yuan XQ Li GC: The emerging landscape of circular RNA ciRS-7 in cancer (Review). Oncol Rep 2015;33:2669-2674.

15 Li J, Lin H, Sun Z, Kong G, Yan X, Wang Y, Wang X, Wen Y, Liu X, Zheng H, Jia M, Shi Z, Xu R, Yang S, Yuan F: High-Throughput Data of Circular RNA Profiles in Human Temporal Cortex Tissue Reveals Novel Insights into Temporal Lobe Epilepsy. Cell Physiol Biochem 2018;45:677-691.

16 Han D, Li J, Wang H, Su X, Hou J, Gu Y, Qian C, Lin Y, Liu X, Huang M, Li N, Zhou W, Yu Y, Cao X: Circular RNA circMT01 acts as the sponge of microRNA-9 to suppress hepatocellular carcinoma progression. Hepatology 2017;66:1151-1164.

17 Zhong Z, Huang M, Lv M, He Y, Duan C, Zhang L, Chen J: Circular RNA MYLK as a competing endogenous RNA promotes bladder cancer progression through modulating VEGFA/VEGFR2 signaling pathway. Cancer Lett 2017;403:305-317.

18 Huang LG, Zou J, Lu QC: Silencing rno-miR-155-5p in rat temporal lobe epilepsy model reduces pathophysiological features and cell apoptosis by activating Sestrin-3. Brain Res 2018;1689:109-122.

19 Dombkowski AA, Batista CE, Cukovic D, Carruthers NJ, Ranganathan R, Shukla U, Stemmer PM, Chugani HT, Chugani DC: Cortical Tubers: Windows into Dysregulation of Epilepsy Risk and Synaptic Signaling Genes by MicroRNAs. Cereb Cortex 2016;26:1059-1071. 